\title{
Successful Treatment of Airplane Headache with Rizatriptan: Case Report
}

\author{
Iryna Delva Mykhaylo Delva \\ Department of Neurological Diseases with Neurosurgery and Medical Genetics, Ukrainian \\ Medical Stomatological Academy, Poltava, Ukraine
}

\section{Keywords}

Airplane headache $\cdot$ Take-off · Landing $\cdot$ Flight duration $\cdot$ Rizatriptan

\begin{abstract}
Optimal management of airplane headache $(\mathrm{AH})$ is still unresolved. A female, 53 years, complained of severe short-lasting jabbing pain attacks over the forehead and in the eyebrows, mainly on the left side, that occur during take-off and landing. Neurological, opthalmological, and otolaryngological examinations and brain MRI were normal. It was diagnosed as $\mathrm{AH}$. The patient was recommended to take $10 \mathrm{mg}$ rizatriptan $30 \mathrm{~min}$ before the flight. It resulted in a complete absence of headache during the take-off and significant decrease of pain intensity during the plane descending. Based on the flight duration (about $2.5 \mathrm{~h}$ ) and rizatriptan pharmacokinetics, the patient was recommended to take a second dose of rizatriptan $10 \mathrm{mg} 1 \mathrm{~h}$ before flight ending. The patient reported a complete absence of pain attacks during the next flights. The effectiveness of $\mathrm{AH}$ pain attack prevention is based on the pharmacokinetic properties of the drug, time of pain onset during flight, and the flight duration.
\end{abstract}

\section{(C) 2021 The Author(s).}

Published by S. Karger AG, Basel

\section{Introduction}

Today, in Ukraine and likely in most of the post-Soviet countries, proper management of headaches, especially rare headaches, remains a major problem [1]. Airplane headache (AH) is a rare form of headache disorder that develops exclusively during plane travels. AH was first reported in 2004 [2], and since then, there has been a steady increase in the frequency of reported cases. AH severe pain intensity can cause fear of flying and other anxious symptoms. Up to now, no specific treatment plan has been developed for $\mathrm{AH}$, although several medications have shown beneficial effects (nonsteroidal anti-inflammatory drugs and 
triptans) $[3,4]$. Here, we report the case of successful treatment of AH pain attacks during the take-off and during the plane descending by rizatriptan if the drug was taken in certain time points based on the flight duration.

\section{Case}

A patient (53 years old, female, school teacher) presented to a primary headache center at the Department of Neurological Diseases with Neurosurgery and Medical Genetics of Ukrainian Medical Stomatological Academy (Poltava city).

The patient complained of headache attacks that occur during each flight. Such headaches never occurred outside of plane flights. For family reasons, the patient regularly flies the same flight to Germany and back 2-3 times a year. The patient had the same headache episodes that occur during take-off and during plane descending. As a rule, a few minutes after the flight take-off, the patient suddenly experiences severe jabbing pain over the forehead and in the eyebrows, mainly on the left side (like thousands of needles are piercing through the head). Pain intensity was assessed retrospectively on visit at 10 points on the visual analog scale (VAS) of $0-10$ with 0 representing no pain and 10 representing worst possible pain. There were no associated auras, photophobia, phonophobia, nausea, vomiting, or trigeminal autonomic symptoms (nasal congestion, tearing, and conjunctival injection). Pain attacks lasted no longer than 15-20 min. Also, the same pain attacks started during plane descending 10-15 min before the landing and always self-resolved when the patient was still on the plane. Rubbing forehead, swinging on the seat, and deeply breathing caused slight pain relief. As a rule, for the whole next day after flight, the patient experienced heavy head feeling.

The first headache attack occurred 3 years before for no apparent reason during plane descending. The patient was very frightened and after arrival was examined by an airport doctor in her passenger seat. Subsequently, the same pain occurred during every next flight.

More than once, the patient sought medical care for flight headache. The patient was examined by 2 outpatient neurologists, and no pathology was found. The patient without prescriptions tried to take before and during the flight ibuprofen $400 \mathrm{mg}$ - without effect, as well as a combined analgesic drug "Citramon" (acetylsalicylic acid $240 \mathrm{mg}$, paracetamol 180 $\mathrm{mg}$, and caffeine $30 \mathrm{mg}$ ) - also without the slightest effect.

The patient denied the history of migraine. The patient had frequent episodic tensiontype headache (2-3 points on VAS) 1-2 times per month that were provoked by mental stresses. She has not taken any medication for tension-type headache relief. She does not have chronic diseases, including arterial hypertension and other cardiovascular disorders.

On patient examination, arterial pressure 125/80 mm Hg and pulse rhythmic 72 beats per minute were obtained. The neurological examination was without any abnormalities. The Hospital Anxiety and Depression Scale was 4 for anxiety and 5 for depression. Otolaryngologist, ophthalmologist, and therapist consultations did not reveal any abnormalities.

The blood tests, including the complete blood count, erythrocyte sedimentation rate, electrolytes, C-reactive protein, biochemical liver, kidney tests, and fasting glucose levels, were normal. A 12-lead electrocardiogram was normal. Brain MRI was also normal. According to the International Classification Headache Disorders-3 criteria [5], we diagnosed AH.

Due to ineffectiveness of nonsteroidal analgesic drugs, the patient was recommended to take $10 \mathrm{mg}$ rizatriptan $30 \mathrm{~min}$ before the flight. After the next flight, by phone, the patient reported a complete absence of headache during the take-off and significant decrease of pain intensity (to 3-4 points on VAS) during plane descending. Based on the flight duration (about $2.5 \mathrm{~h}$ ) and rizatriptan pharmacokinetics (the time of maximum plasma concentration is $1 \mathrm{~h}$ and the time of half-life is $2-3 \mathrm{~h}$ [6]) in order to prevent pain attack also during the descending

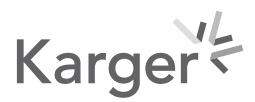


phase, the patient was recommended to take a second dose of rizatriptan $10 \mathrm{mg} 1 \mathrm{~h}$ before flight ending (thus, $2 \mathrm{~h}$ after the first rizatriptan dose).

At the next visit, the patient reported a complete absence of pain attacks during the take-off as well as during the landing. It has been recommended to use rizatriptan $10 \mathrm{mg}$ according to the special intake regimen (in $30 \mathrm{~min}$ before the take-off and repeatedly $1 \mathrm{~h}$ before the flight ending) on the same flights or on flights with longer duration.

\section{Discussion}

Up to now, there is still uncertainty about the treatment of $\mathrm{AH}$. The current literature indicates that $\mathrm{AH}$ can be treated with simple analgesics and nonsteroidal anti-inflammatory drugs (such as naproxen or ibuprofen) administered before airplane travel [3]. But, in our patient, the use of ibuprofen and combined analgesic drug had no effects.

Also, the current literature indicates that triptans might be effective for $\mathrm{AH}[3,4]$. To this day, effects of triptans on $\mathrm{AH}$ pain are hypothetical. One assumption is that $\mathrm{AH}$ might be caused by a rapidly changing cabin pressure, as it is seen during take-off and landing phases $[3,7,8]$. Due to possible variations in the anatomical and structural construction in the individual ethmoid sinuses (ethmoid sinuses have random pneumatization patterns that vary among individuals), these patients cannot equalize the pressure during the take-off or landing [8]. Ethmoid cells and ethmoidal arteries are innervated by the ethmoidal nerve (ophthalmic branch of the trigeminal nerve), where ethmoidal nerve endings may trigger a stimulus as a consequence of sinus barotrauma and thereby local inflammation in the tissue due to the lack of pressure equalization thus activating the trigeminal vascular system as well as trigeminal cervical complex [3, 4, 7-11]. Prostaglandin E2 has been shown to be significantly high in $\mathrm{AH}$ patients during a simulated flight in a pressure chamber when compared with healthy subjects [9]. It is speculated that prostaglandin E2 is elevated due to local inflammation, which may cause vasodilation in the cerebral arteries and induce $\mathrm{AH}$ [9]. So, it has been suggested that triptans prevent $\mathrm{AH}$ attacks through multiple mechanisms of action - vasoconstriction effect, peripheral neuronal inhibition, inhibition of transmission through second-order neurons of the trigeminocervical complex, and inhibitory influences on trigeminovascular sensory input $[8,9]$.

Up to now, it remains unclear which of the triptans should be chosen to prevent AH. Ipekdal et al. [8] reported clinical cases of complete prevention of AH by different triptans (sumatriptan, zolmitriptan, eletriptan, and naratriptan). As for rizatriptan, there is only 1 report on prescription of rizatriptan $(10 \mathrm{mg})$ for $\mathrm{AH}$ with effective prevention of $\mathrm{AH}$ attacks [12]. Among the 4 triptans (sumatriptan, zolmitriptan, rizatriptan, and eletriptan) that were available in our country at the time of patient visit, we selected rizatriptan. All mentioned triptans (except sumatriptan) have almost similar availability (40-50\%); however, rizatriptan has the shortest time of maximum plasma concentration (just over $1 \mathrm{~h}$ ) [13]. The latter advantage of rizatriptan makes it possible to take the drug comparatively shortly before the flight and thus taking into account the possible flight delay. From theoretical point of view, in our case (flight duration about $2.5 \mathrm{~h}$ ) for prevention of landing $\mathrm{AH}$, it is appropriate to use triptan with longer half-life time than rizatriptan (e.g., eletriptan 3.5-6 h [13]). But, we decided not to change the drug because of good effectiveness of rizatriptan for prevention of pain attack during the ascending phase.

Earlier it was assumed that prophylactic drug management of AH should be appropriately timed depending on the time of $\mathrm{AH}$ occurrence (during take-off, landing, or both), depending on the duration of air travel, and depending on the peak action of prophylactic medication $[8,12]$. According to a systemic review, onset of $\mathrm{AH}$ was found mostly during the

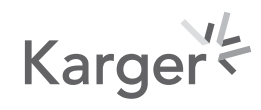


descending phase in 210 patients $(n=210 / 275)$, followed by the ascending phase in 33 patients $(n=33 / 275)$, and only 18 patients $(n=18 / 275)$ were found to report it both during descending and ascending phases [4].

The simplest regimen of triptan use is in the cases of $\mathrm{AH}$ pain occurrence only during the take-off - triptans are reported to be the most effective when taken $30 \mathrm{~min}$ before a flight [8, 12]. In cases of $\mathrm{AH}$ pain occurrence during landing or even during both take-off and landing, it needs to be taken into account also the flight duration and drug pharmacokinetics. It had been shown that patients with AH during landing took 1 regular tablet of rizatriptan $30 \mathrm{~min}$ before the flight and subsequently became completely AH free in short-lasting flights (1-2 h), but experienced a mild headache in long-lasting flights (2-3 h) [12]. The latter phenomenon was explained by the pharmacokinetics of rizatriptan [6].

When prescribing rizatriptan $30 \mathrm{~min}$ before the flight, we used the experience of successful use of triptans about $30 \mathrm{~min}$ before the flight for prevention of pain attacks during ascending $[8,12]$. Most likely, based on the flight duration (time period between rizatriptan intake before the take-off and time of $\mathrm{AH}$ pain onset during the plane descending was a little over $2.5 \mathrm{~h}$ ), rizatriptan blood concentration in our patients at the time of descending had already decreased to a critical level, insufficient to prevent AH pain attack during the descending phase. Based on the chronology (interval between taking the first dose of rizatriptan and pain onset during the ascending phase is about $40-45 \mathrm{~min}$ ), for $\mathrm{AH}$ attack prevention at the end of flight, we were guided by the same logic - the time interval between taking the second dose of rizatriptan and pain onset during the descending phase should also be about $45 \mathrm{~min}$. So, it was recommended to take rizatriptan just $1 \mathrm{~h}$ before flight ending (which is approximately $45 \mathrm{~min}$ before the expected onset of pain). So, in cases of AH pain attacks during both take-off and descending, if a flight duration is $2 \mathrm{~h}$ or more, it is likely expedient to take the first dose of rizatriptan $10 \mathrm{mg}$ in $30 \mathrm{~min}$ before take-off and the second dose of the drug $10 \mathrm{mg}$ in $1 \mathrm{~h}$ before flight ending. However, most likely, this issue is very individual and requires further clarification. The main limitation of the study is its single-case study content of AH treated with rizatriptan, so we cannot confidently exclude a placebo effect.

\section{Conclusion}

In cases of AH attack occurrence during both take-off and landing, for effective use of rizatriptan, flight duration should be taken into account, as well as the pharmacokinetic properties of the drug.

\section{Statement of Ethics}

Written informed consent was obtained from the patient for publication of this case report and any accompanying images. The study has been approved by the Ethics Committee at Ukrainian Medical Stomatological Academy and has therefore been performed in accordance with the ethical standards laid down in the 1964 Declaration of Helsinki and its later amendments.

\section{Conflict of Interest Statement}

The authors do not have any conflicts of interest to declare.

\section{Karger'}




\section{Case Reports in Neurology}

\section{Funding Sources}

The authors have not received any funding for conduct, authorship, or publication of this study.

\section{Author Contributions}

Iryna Delva involved in data acquisition, case management, and manuscript preparation. Mykhaylo Delva involved in concept, study design, definition of intellectual content, data analysis, manuscript preparation, manuscript editing, and manuscript review.

\section{References}

1 Delva M, Delva I. Analysis of primary headaches management in poltava regions. Wiad Lek. 2021 Jan;74(1): 118-21.

2 Atkinson V, Lee L. An unusual case of an airplane headache. Headache. 2004 May;44(5):438-9.

3 Berilgen MS, Müngen B. A new type of headache, headache associated with airplane travel: preliminary diagnostic criteria and possible mechanisms of aetiopathogenesis. Cephalalgia. 2011 Jul;31(12):1266-73.

4 Bui SBD, Gazerani P. Headache attributed to airplane travel: diagnosis, pathophysiology, and treatment: a systematic review. J Headache Pain. 2017 Aug;18(1):84.

5 Olesen J. Headache classification committee of the international headache society (IHS) the international classification of headache disorders, abstracts. Cephalalgia. 2018 Jan;38(1):1-211.

6 Smet D. Pharmacokinetics and tolerability of oral rizatriptan in healthy male and female volunteers. Br J Clin Pharmacol. 1999;47(4):373-8.

7 Bui SB, Petersen T, Poulsen JN, Gazerani P. Headaches attributed to airplane travel: a Danish survey. J Headache Pain. 2016 Apr;17(1):33.

8 Ipekdal HI, Karadaş Ö, Öz O, Ulaş ÜH. Can triptans safely be used for airplane headache? Neurol Sci. 2011 May; 32(6):1165-9.

9 Bui SBD, Petersen T, Poulsen JN, Gazerani P. Simulated airplane headache: a proxy towards identification of underlying mechanisms. J Headache Pain. 2017 Jan;18(1):9.

10 Mainardi F, Maggioni F, Lisotto C, Zanchin G. Diagnosis and management of headache attributed to airplane travel. Curr Neurol Neurosci Rep. 2013 Jan;13(3):335.

11 Kararizou E, Anagnostou E, Paraskevas GP, Vassilopoulou SD, Naoumis D, Kararizos G, et al. Headache during airplane travel ("airplane headache"): first case in Greece. J Headache Pain. 2011 Apr;12(4):489-91.

12 Bui SBD, Antropova O, Gazerani P. An anecdotal case of treatment of headache attributed to airplane travel: are triptans an option? SN Compr Clin Med. 2019 May;1(7):527-8.

13 Rapoport AM, Tepper SJ. All triptans are not the same. J Headache Pain. 2001 Sep;2(1):87-92.

\section{Karger'}

